\title{
Ultra-High Specific Absorption of Metallic Binary-Groove Gratings
}

\author{
Yutong Li, Yumin Liu \\ State Key Laboratory of Information Photonics and Optical Communications, Beijing University of Posts and \\ Telecommunications, Beijing, China \\ Email: lytbupt@163.com
}

How to cite this paper: Li, Y.T. and Liu, Y.M. (2017) Ultra-High Specific Absorption of Metallic Binary-Groove Gratings. Optics and Photonics Journal, 7, 92-98. https://doi.org/10.4236/opj.2017.78B013

Received: June 27, 2017

Accepted: August 7, 2017

Published: August 10, 2017

\begin{abstract}
A metallic binary-groove gratings, which can be exploited to absorb light at C-band and sense materials with refractive index (1.0 - 1.2), is proposed and investigated numerically. Both ultra-high absorption (99.937\%) and sensitivity $(1600 \mathrm{~nm} / \mathrm{RIU})$ are achieved.
\end{abstract}

\section{Keywords}

Surface Plasmon, Absorption, Gratings, Sensing

\section{Introduction}

The surface plasmon polaritons (SPPs), which is an evanescent wave propagating along metal-insulator interface with extremely confined mode area, can be effectively excited by an applied electromagnetic wave. Recently, a great many of the SPPs-based devices have been extensively designed for applications in nanophotonic circuits, optical communication, solar cells and bio-sensors, etc. The structure for SPPs exciting has been a hot topic in last few decades. Among the investigations, periodic metallic gratings are considered as one of the common structures for transferring the energy of incident wave to SPPs. Much related work has been done to investigate effective index, propagation length and energy absorption [1] [2] [3]. However, the present results mainly focus on theoretical features rather than practical applications.

In this paper, a structure of periodic binary grooves is proposed. Due to the coupling of the modes in two grooves, complete absorption peak of an incident wavelength at C-band (1550 nm here) is realized by silver-based structure. The required wavelength can be manipulated by varying optical properties of the structure and materials (silver, gold, aluminum). In addition, the sensing cha- 
racteristic is also calculated about the proposed structure. We use commercial software COMSOL Multiphysics 5.2.

\section{Model Structure}

The resonant absorption behavior is strongly depending on geometric parameters, including width and depth of grooves and spacing distance [4] [5]. The two grooves separated from each other with spacing a have and $\mathrm{w}_{2}$ in width, $\mathrm{d}_{1}$ and $\mathrm{d}_{2}$ in depth, respectively. Considering designing a perfect absorber, we set the thickness of $\mathrm{Ag}$ as $800 \mathrm{~nm}$ that is enough for blocking light transmission. Notice that, the whole structure is considered to be infinite in $\mathrm{z}$-axis and solved by finite element method (FEM) in our simulation. We use metal silver first because of its low optical loss and excellent plasmonic behavior. The electric permittivity of Ag is given by the following (1) Drude model,

$$
\varepsilon_{\mathrm{m}}=\varepsilon_{\infty}-\omega_{\mathrm{p}}^{2} /\left(\omega^{2}+\mathrm{i} * \gamma * \omega\right)
$$

where $\omega$ is the angular frequency, $\omega_{\mathrm{p}}=9.1 \mathrm{eV}$ is the plasma frequency, $\varepsilon_{\infty}=3.7$ is a corrective constant that accounts for the background electron screening at high frequency, and $\gamma=0.018 \mathrm{eV}$ is the electron scattering frequency.

\section{Simulation Results}

Figure 2 shows the calculated spectral properties of reflectance, transmittance and absorbance for the AS structure. In addition, perfect narrow band absorption occurs at $1550 \mathrm{~nm}$. The two grooves in one period are designed with different values of depth, so that two different cavity modes can be created for coupling. The absorbance is defined as

$$
A(\omega)=1-T(\omega)-R(\omega)=1-\left|S_{11}\right|^{2}-\left|S_{21}\right|^{2}
$$

where $T(\omega)=\left|S_{21}\right|^{2}$ and $R(\omega)=\left|S_{11}\right|^{2}$ are transmittance and reflectance, respectively. The distributions of normalized electric field and power flow obtained by silver-based gratings are shown in Figure 2(b). As we can see, the strongest localized field appears at the outer edge corners and their spacing region. The incident TM-polarized wave is efficiently coupled into both grooves.

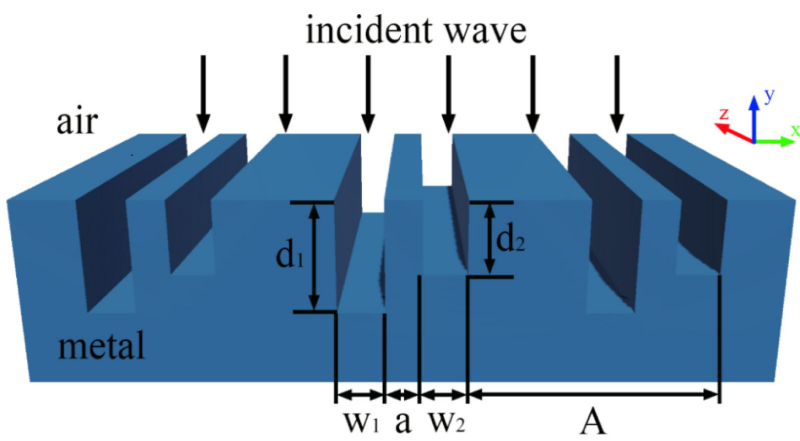

Figure 1. The schematic diagram of the proposed binary grooves-based metallic gratings. 


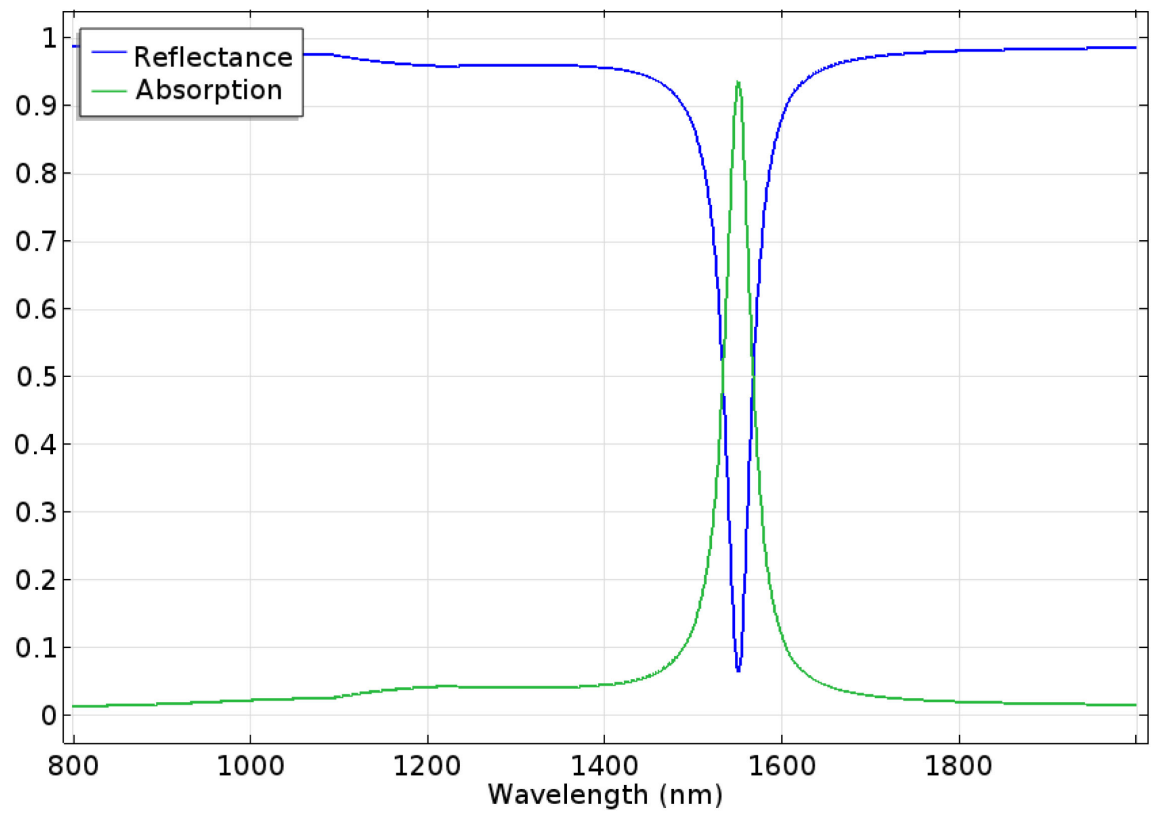

(a)

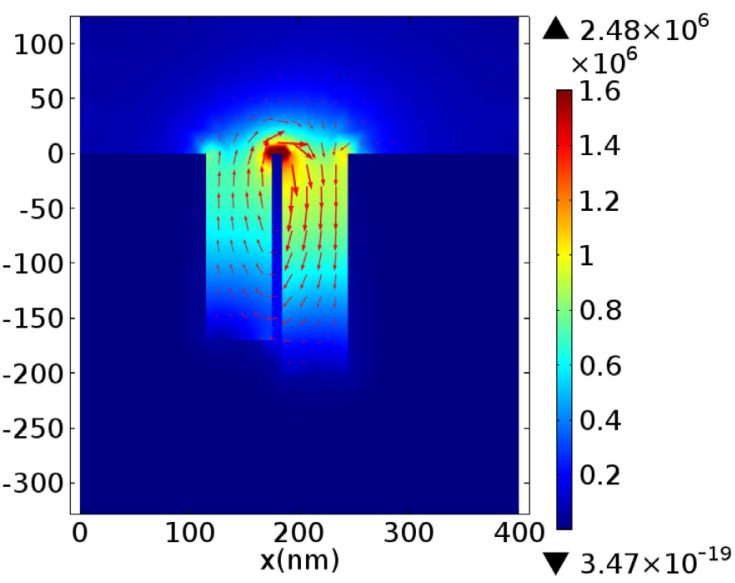

(b)

Figure 2. (a) Calculated reflectivity, transmission and absorption spectra of the AS structure; (b) The normalized electric field and power flow of the metallic gratings.

Except for the C-band absorber at $1550 \mathrm{~nm}$, another application of the proposed structure is sensing refractive index of material under detecting. As is shown in Figure 3(a), five resonant peaks are presented with refractive index changing from 1.0 to 1.2 (an increment of 0.05 ). Notice that, the materials are filled in the whole region except metal. After calculation, the sensitivity about $1600 \mathrm{~nm} / \mathrm{RIU}$ is achieved.

As it depicts in Figure 3(b), with the refractive index changed from 1.1 to 1.2, the peak has a red shift from about $1550 \mathrm{~nm}$ to $1870 \mathrm{~nm}$. The metallic binarygroove structure has a favored linear characteristic. The full wave at half maximum (FWHM) of the peaks are getting wider with the increasing refractive index which can be seen in Figure 3(c). 


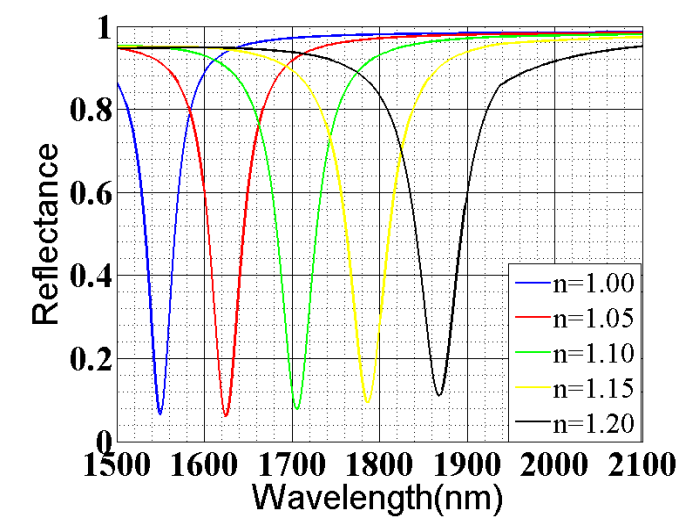

(a)

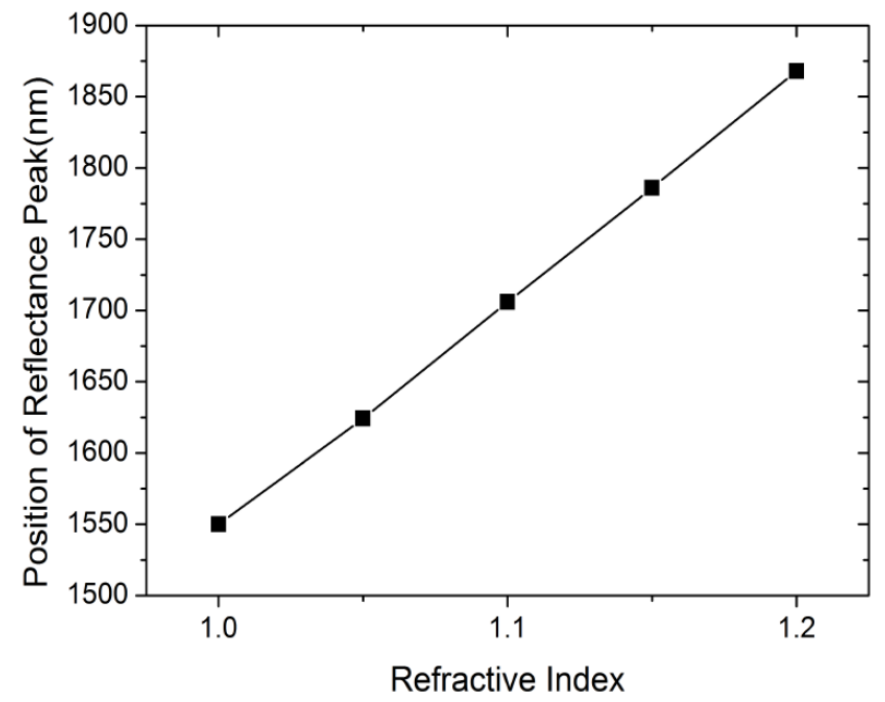

(b)

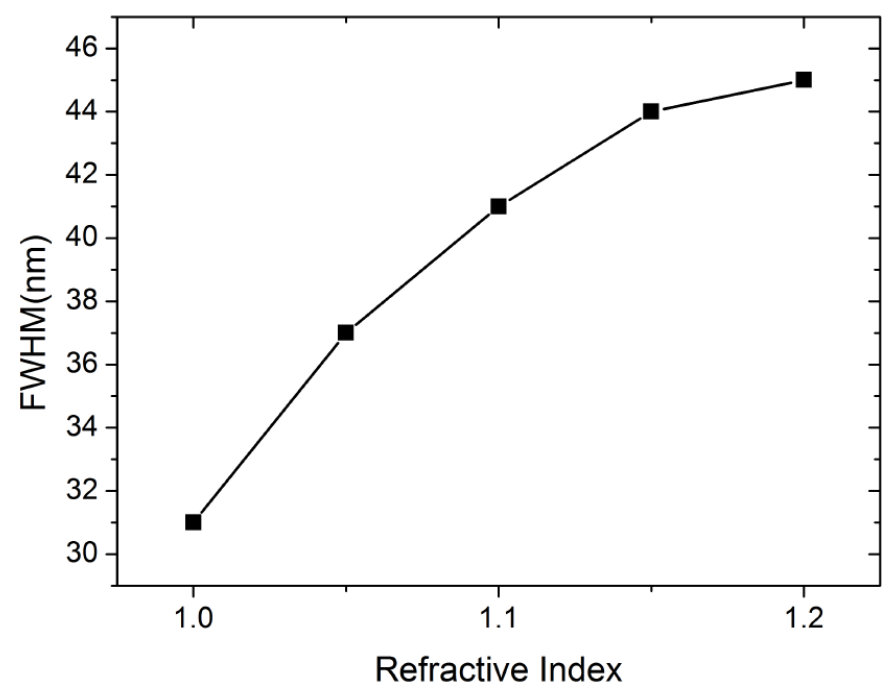

(c)

Figure 3. (a) The reflectance spectrum versus incident wavelength for refractive index varying from 1.00 to 1.20; (b) The linear relation of peak position; (c) The FWHM versus refractive index. 


\section{Analysis and Applications}

The study on the optical properties of the absorber is investigated. The reflectance dip varied with different $\mathrm{w}$ which means the best absorber can be fixed at a value of 1111. The depths of two grooves have effect on the interaction which finally influence the absorption rate at a certain wavelength, such as $1550 \mathrm{~nm}$. Besides, the absorber should work over a wide range of incident angle for practical application. The absorption property of our plasmonic absorber with the same geometric parameters as Figure 3 in terms of the indent angle ranging from $0^{\circ}$ to $40^{\circ}$ for TM polarization was investigated by utilizing FEM, and the corresponding absorption spectra was illustrated in Figure 4. It is found that the absorption spectrum is nearly insensitive to the change of the incident angle. This can be explained in this way: for the TM polarization, the magnetic field of the incident light is always perpendicular to the incident plane with various incident angles and it can efficiently drive the circulating currents at large angles of incidence. Therefore, the absorption peak is nearly independent of the incident angle and it keeps more than $97 \%$ even at $40^{\circ}$. In addition, the comparison of different metals is necessary in practical application. Firstly, metals can give rise to different absorption behaviors of the structures due to their different relative dielectric constants [6]. Secondly, the fabrication cost of metal is another important factor should be taken into consideration. Therefore, metals of silver, gold and aluminum are compared when we trying to obtain the reflectance dip (minimum) at wavelength $1550 \mathrm{~nm}$. In order to ensure full absorption at 1550 $\mathrm{nm}$ under these kinds of metal, corresponding structural parameters (a, $\mathrm{d}$ and $\mathrm{w}$ ) are optimized.

The reflectance spectrums obtained by the silver, gold and aluminum-based structure are shown in Figures 5(a)-(c), respectively. As it can be seen clearly, both silver and gold ensure a precise reflectance minimum of 0.08 and 0.063 at

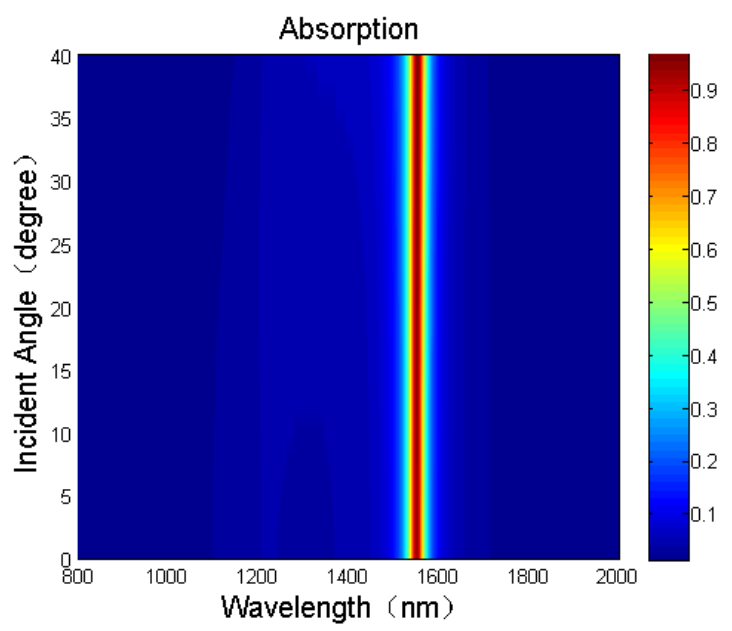

Figure 4. The absorption property of our plasmonic absorber as a function of wavelength for various incident angles for TM polarization. The geometric parameters are the same as those in Figure 1. 


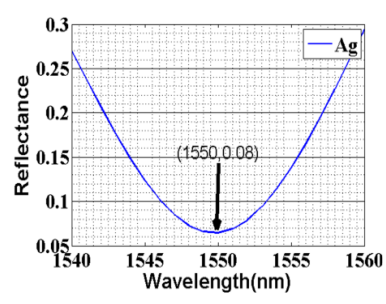

(a)

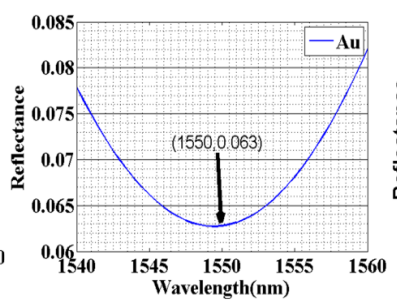

(b)

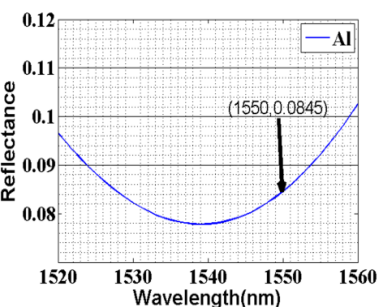

(c)

Figure 5. The reflectance of the structure with different type of metal. (a) silver; (b) gold and (c) aluminum. The electric field of the structure when the metal is silver. (a) $d_{1}=$ $200 \mathrm{~nm}, \mathrm{~d}_{2}=170 \mathrm{~nm}, \mathrm{w}_{1}=\mathrm{w}_{2}=60 \mathrm{~nm} ; \mathrm{a}=9.25 \mathrm{~nm}$; (b) $\mathrm{d}_{1}=200 \mathrm{~nm}, \mathrm{~d}_{2}=130 \mathrm{~nm}$, $\mathrm{w}_{1}=\mathrm{w}_{2}=60 \mathrm{~nm} ; \mathrm{a}=6.05 \mathrm{~nm} ;(\mathrm{c}) \mathrm{d}_{1}=240 \mathrm{~nm}, \mathrm{~d}_{2}=160 \mathrm{~nm}, \mathrm{w}_{1}=\mathrm{w}_{2}=60 \mathrm{~nm} ; \mathrm{a}$ $=4.9 \mathrm{~nm}$.

$1550 \mathrm{~nm}$, respectively. When the metal of aluminum is used, a reflectance minimum of 0.0845 is obtained. In the meantime, another minimum of 0.078 is obtained at $1539 \mathrm{~nm}$. In general, a reflectance minimum of 0.063 , or an absorption maximum of $99.937 \%$, obtained by gold is the most desired value, despite the metal fabrication cost.

Based on the above analysis, the structures made by gold and aluminum show better robustness than by silver. Regardless of the fabrication cost in practical, gold is the first choice. However, aluminum becomes an alternative for its low production cost.

\section{Conclusion}

In a summary, we propose and investigate metallic binary-groove gratings, which can achieve ultra-high absorption at C-band with silver, gold and aluminum and is nearly independent of the incident angle. As the most commonly used operating wavelength in optical communication, $1550 \mathrm{~nm}$ is captured more precisely by the gold gratings. Besides, the robustness of our structure is analyzed by calculating the error tolerance rate. At last, another application acting as a refractive index sensor is proposed and analyzed.

\section{Acknowledgements}

This work was supported by the national natural science foundation of China (Grants No. 61275201 and No. 61372037).

\section{References}

[1] Bozhevolnyi, S.I., Volkov, V.S., Devaux, E. and Ebbesen, T.W. (2005) Channel Plasmon-Polariton Guiding by Subwavelength Metal Grooves. Physical Review Letters, 95, 046802. https://doi.org/10.1103/PhysRevLett.95.046802

[2] Blake, A. and Sukharev, M. (2015) Surface Plasmon Polaritons in Periodic Arrays of V-Shaped Grooves Strongly Coupled to Quantum Emitters. Physical Review B, 92, 035433. https://doi.org/10.1103/PhysRevB.92.035433

[3] Tan, W.-C., Preist, T.W., Sambles, J.R. and Wanstall, N.P. (1998) Flat Surface- 
Plasmon-Polariton Bands and Resonant Optical Absorption on Short-Pitch Metal Gratings. Physical Review B, 59, 12661-12666.

[4] Le Perchec, J., Quémerais, P., Barbara, A. and López-Ríos, T. (2008) Why Metallic Surfaces with Grooves a Few Nanometers Deep and Wide May Strongly Absorb Visible Light. Physical Review Letters, 100, 066408. https://doi.org/10.1103/PhysRevLett.100.066408

[5] Guo, L., Lyu, M. and Sun, Z. (2015) Full Resonant Absorption of Light in Metallic Subwavelength Gratings with Asymmetric Binary Nanogrooves. Plasmonics, 10, 305-309. https://doi.org/10.1007/s11468-014-9809-x

[6] Novotny, L. and Hecht, B. (2012) Priciples of Nano-Optics. Cambridge University. https://doi.org/10.1017/CBO9780511794193

Submit or recommend next manuscript to SCIRP and we will provide best service for you:

Accepting pre-submission inquiries through Email, Facebook, LinkedIn, Twitter, etc. A wide selection of journals (inclusive of 9 subjects, more than 200 journals)

Providing 24-hour high-quality service

User-friendly online submission system

Fair and swift peer-review system

Efficient typesetting and proofreading procedure

Display of the result of downloads and visits, as well as the number of cited articles

Maximum dissemination of your research work

Submit your manuscript at: http://papersubmission.scirp.org/

Or contactopj@scirp.org 